\title{
Seguridad personal y protección del medio ambiente en el turismo de naturaleza en Colombia ${ }^{1}$
}

Álvaro Gómez Peña ${ }^{2}$

Escuela de Policía en Protección y Seguridad

Resumen. Este capítulo de libro identifica los factores críticos del turismo de naturaleza y aventura en el Departamento de Cundinamarca, Colombia, con el fin de que las autoridades desplieguen acciones que garanticen a los turistas la seguridad personal y el cuidado de los escenarios naturales. Aplicando un método de enfoque mixto de tipo descriptivo, la investigación propone estrategias de prevención y control policial, en materia de protección ambiental y seguridad personal, que permitan proteger a los turistas y disminuir la afectación del medio ambiente como consecuencia del desarrollo de actividades de naturaleza y aventura. Se concluye que es necesaria una pronta articulación interinstitucional entre la Policía Nacional y las entidades del Estado para que desarrollen las acciones propuestas, las cuales estarán contenidas en una guía de actuación policial para el servicio.

Palabras clave: Cundinamarca; información turística; medio ambiente; medida de protección; policía; seguridad; turismo.

1 Este capítulo forma parte de los resultados del proyecto de investigación "Guía para la seguridad personal y protección del medio ambiente en el turismo de naturaleza y aventura en Colombia. Caso Departamento de Cundinamarca", del Grupo de Investigación Escuela de Policía en Protección y Seguridad, perteneciente a la Institución Universitaria Dirección Nacional de Escuelas, Policía Nacional de Colombia, registrado con el código COL0171306 de Minciencias. Los puntos de vista y los resultados presentados en este capítulo pertenecen al autor y no reflejan necesariamente los de las instituciones participantes.

2 Intendente Jefe de la Policía Nacional de Colombia. Magíster en Docencia e Investigación Universitaria de la Universidad Sergio Arboleda de Bogotá. Licenciado en Filosofía de la Universidad Santo Tomás. Docente de planta y líder del Grupo de Investigación de la Escuela de Policía en Protección y Seguridad, Bogotá, D. C., Colombia. OrciD: https://orcid.org/0000-0001-5400-6692 - Contacto: alvaro. gomez6667@policia.edu.co 


\section{Introducción}

La creciente demanda de turistas que practican el turismo de naturaleza y aventura, como también la proliferación de promotores turísticos que no cumplen con las normas estipuladas para regular la práctica de estas actividades, hacen necesario que la Policía Nacional y la Dirección de Protección y Servicios Especiales implementen una serie de acciones de prevención y control policial, en materia de seguridad personal y protección ambiental, que permitan la protección de los turistas y la disminución a la afectación del medio ambiente.

La Policía Nacional de Colombia tiene la responsabilidad de generar acciones que garanticen a los practicantes del turismo de naturaleza y aventura la seguridad personal, buscando que no tengan temor mediante la adopción de patrones individuales de percepción del peligro, la adopción de conductas de prevención (previsión, predicción, preparación) ante los riesgos, medidas de protección (disuasión, reacción) frente a la amenaza o la incertidumbre y la preservación (conservación de la vida o recuperación de la lesión) (La Rotta, 2005, p. 571). Por consiguiente, este trabajo se plantea la siguiente pregunta de investigación: ¿Cómo mejorar la seguridad personal y ambiental, debido al turismo de naturaleza y aventura, en el Departamento de Cundinamarca, por parte del Área de Turismo, de la Dirección de Protección y Servicios Especiales?

En la actualidad no existe un elemento doctrinal en la Policía Nacional que permita orientar a los hombres y las mujeres que cumplen funciones como policías de turismo sobre los procedimientos que deben desarrollar, en relación con el turismo de naturaleza y aventura. Como resultado de esta investigación se diseñó una guía de actuación policial, que contiene las estrategias que debe implementar la Policía Nacional para garantizar la seguridad personal y ambiental en el turismo de naturaleza y aventura. Puntualmente, se identifican los factores críticos que se presentan y que requieren la intervención de la Policía Nacional, y se caracterizan las acciones específicas para la protección ambiental y la seguridad personal que permitan la prevención y control policial. 


\section{Marco teórico}

\section{Seguridad}

El término seguridad hace alusión a la obligación de preocuparse por todas las circunstancias que rodean a las personas. Ballesteros (2004) sostiene que "la seguridad en su acepción más radical, significaría el control total de todos los riesgos en todos los ámbitos y sin límite temporal, lo que es absolutamente imposible de conseguir [...] la seguridad queda delimitada por los intereses que protege” (pp. 17-33). Así mismo, Rojas (2011) asevera que "el ideal de la seguridad es mantener en perfecto orden y libre de cualquier riesgo tanto las personas como sus bienes, para ello han de generarse todo tipo de políticas y procedimientos que permitan a todos los ciudadanos tener un ambiente sano, libre de peligro" (pp. 1-2). El mismo autor, citando el diccionario Webster's New Collegiate, define la seguridad como "la cualidad o estado de estar seguro [...] libertad del peligro. Lo seguro [...] libertad de o libre de temor o ansiedad [...] protección [...] medidas tomadas para proteger" (Webster's New Collegiate, 2002, p. 5).

Por su parte, Franco y Jaramillo (2010), citando a Green (s. f.), señalan que "la seguridad, en su sentido semántico y filosófico, implica un ambiente relativamente estable y predecible en el cual un individuo o grupo pueden desarrollar sus objetivos sin interferencia o daño y sin miedo a disturbios o pérdidas" (p. 19). Asimismo, citando a Post y Kingsbury (s. f.), definen la seguridad como "los medios activos o pasivos, que sirven para proteger y preservar un medio que permite desarrollar actividades en una organización o sociedad sin interferencias" (Franco \& Jaramillo, 2010, p. 19).

\section{Seguridad personal}

La seguridad personal establece vínculos personales, da guías de comportamiento y suministra estructuras de apoyo. Por consiguiente, le corresponde a la Policía Nacional generar acciones que permitan que se garantice a los practicantes del turismo de naturaleza y aventura la seguridad personal, entendida como 
la que busca proveer la ausencia de temor mediante patrones individuales de percepción del peligro, adopción de conductas de prevención (previsión, predicción, preparación) ante los riesgos, medidas de protección (disuasión, reacción) frente a la amenaza o la incertidumbre y preservación (conservación de la vida o recuperación de la lesión) ante el ataque. (La Rotta, 2005, p. 571)

\section{Turismo}

"El turismo como fenómeno social y como actividad económica es desde hace unos años una realidad asumida" (Huesear, 1993). La Organización Mundial de Turismo (Омт), en la Conferencia Internacional sobre "Estadísticas de Viajes Turísticos de Ottawa" en 1991, propuso una serie de recomendaciones para el turismo internacional, entre las cuales está "proveer un vínculo entre oferta y demanda turística”. Asimismo, estableció definiciones de las actividades turísticas, como espacio geográfico y operadores turísticos - que podían comprender la totalidad de los consumidores y los servicios que ofrece el turismo-, clasificándolos en viajeros, visitantes, turistas y excursionistas.

Teniendo en cuenta el lugar o la situación de procedencia de los turistas y el destino elegido, la OMT estableció unos nuevos términos: (1) turismo doméstico o interno: visitantes de su propio país; (2) turismo receptivo: procedentes de otro país, y (3) turismo emisor: ciudadanos del propio país y que se dirigen a otros países. Además, se debe tener en cuenta que "el turismo es una rama de la actividad económica que representa aproximadamente el $10 \%$ del Piв mundial y esta participación viene creciendo en los últimos decenios" (Brida et al., 2011, p. 291). Asimismo, uno de los puntos del Código de Ética Mundial para el Turismo, reconoce que el turismo de naturaleza y el ecoturismo son "formas de turismo particularmente enriquecedoras y valorizadoras, siempre que respeten el patrimonio natural y la población local y se ajusten a la capacidad de ocupación de los lugares turísticos" (Organización Mundial del Turismo, 2017).

Finalmente, es importante y de trascendencia conocer las expectativas y las percepciones que tienen los turistas y los habitantes del sitio de esparcimiento, desde el punto de vista de la competitividad turística, en cuanto a la seguridad general del entorno y, consecuentemente, la seguridad personal. En coherencia con este planteamiento, el Plan Estratégico de Seguridad Turística 
tiene como propósito buscar la seguridad turística, entendiéndola como "una de las condiciones fundamentales para el fortalecimiento de la competitividad del turismo en Colombia”, que involucra a la Policía Nacional de Colombia, la Dirección de Protección y Servicios Especiales (Ministerio de Comercio, Industria y Turismo, 2017).

\section{Medio ambiente}

Los beneficios sociales y económicos han hecho que el hombre, con sus acciones, deteriore y cause daño ambiental en los escenarios naturales donde se realiza turismo de naturaleza y aventura. Por consiguiente, pareciera que son pocas las acciones que realiza para la preservación del medio ambiente, pues tiene la falsa concepción de que los recursos naturales pueden regenerarse rápidamente, subsanándose por la naturaleza en su entorno natural. Teniendo presente que la actividad del hombre por lo general tiene intereses en su bienestar y que no propenden a la conservación de ese equilibrio, se ha generado un proceso de degradación medioambiental que, de seguir así, puede llevar a un verdadero caos. Los turistas pueden hacer daños a los recursos naturales, tales como la vegetación y el agua, por el sobreuso o una actividad no controlada. Por esta razón, “con frecuencia se considera la minimización del daño ambiental por el turismo y la maximización de los beneficios sociales para los residentes como dos retos diferentes, por distintos grupos de personas" (Ashley \& Roe, 2002, s. p.).

En el desarrollo del turismo de aventura es evidente el daño a los escenarios naturales para practicar dicha actividad, pues el deterioro causado por el progreso acelerado del turismo en diferentes lugares del país conllevará el deterioro y la destrucción de entornos ecológicos. Por esto se hace necesario de manera urgente que el Viceministerio de Turismo realice los estudios correspondientes, en coordinación con el Ministerio de Medio Ambiente, que permitan, por un lado, crear los mecanismos necesarios para mitigar el impacto ambiental en el desarrollo del turismo en todas sus modalidades $y$, por otro, generar la reglamentación que se requiera con el fin de regular las actividades deportivas y de aventura — que en estos momentos no se encuentran debidamente certificadas como acciones seguras - a través de los protocolos que se establezcan para este fin. 


\section{Métodos ]}

El trabajo investigativo se abordó con un enfoque cualitativo y cuantitativo de tipo descriptivo. La recolección de datos se realizó con el fin de adquirir conocimientos y opiniones de los participantes referente a las medidas de protección personal, autoprotección y medidas de conservación que se pretende implementar en los entornos naturales que permiten el disfrute de los diferentes escenarios donde se presenta turismo de naturaleza y aventura, con visión holística y basados en las experiencias de los individuos, conceptos técnicos sobre su estructura y composición desde la parte social, cultural, económica, ambiental y normativa. Se desarrollaron las variables subjetivas, tales como riesgo, vulnerabilidad e impacto que genera el desarrollo del turismo de aventura, con las cuales se diseñó un mecanismo de control que mitigue la ocurrencia de hechos que afecten la vida e integridad de los turistas, quienes son el principal factor para el desarrollo de escenarios turísticos.

Para la recolección de la información se diseñaron cuatro instrumentos tipo encuesta, pues según Quezada (2010), “el diseño de una encuesta parte de la premisa de que si queremos conocer algo sobre el comportamiento de las personas, lo mejor, lo más directo y simple, es preguntárselo directamente a ellas" (p. 124). La primera se aplicó al 83,33 \% del universo de los policías adscritos al Grupo de Protección al Turismo y Patrimonio Nacional de la Seccional de Protección y Servicios Especiales del Departamento de Cundinamarca. El segundo instrumento tipo encuesta fue aplicado a las autoridades locales (alcaldes, secretarios de Gobierno y/o Cultura y Turismo de los municipios). El tercer instrumento diseñado fue aplicado a los operadores turísticos, que prestan el servicio de planes de turismo de naturaleza y aventura. El último instrumento aplicado tuvo como población objeto los turistas que realizan turismo de naturaleza y aventura. Las encuestas fueron validadas por expertos temáticos y el análisis de la información se realizó utilizando el programa estadístico informático Spss.

Finalmente, también se realizaron actividades concernientes al ingreso de diferentes bases de datos de revistas indexadas, portales o páginas web con el fin de obtener información. De igual forma, se llevaron a cabo visitas 
in situ a la Dirección de Protección y Servicios Especiales, al Departamento de Policía de Cundinamarca y al Viceministerio de Turismo en Colombia, y se recolectó información sobre las acciones necesarias para generar herramientas que permitan regular, controlar y sancionar.

\section{Resultados}

A continuación, se presentan las estrategias de prevención y control policial propuestas en materia de protección ambiental y seguridad personal para la protección de los turistas y la disminución a la afectación del medio ambiente como consecuencia del desarrollo de actividades de naturaleza y aventura. Específicamente, se hace referencia a las estrategias que debe aplicar el Área de Turismo de la Dirección de Protección y Servicios Especiales y que estén contenidas en la guía de actuación policial:

1. Realizar campañas de difusión de la normatividad vigente a los prestadores de servicios de turismo de naturaleza y aventura formales e informales. En ellas se aclaran los requisitos a los que están obligados y las sanciones legales a las que se pueden ver expuestos en caso de no cumplir.

2. Realizar mesas de trabajo con los operadores turísticos con el fin de diseñar planes de prevención y manejo de riesgos para cada actividad que ofrecen de turismo de naturaleza y aventura en los entornos ecológicos.

3. Diseñar políticas y planes de responsabilidad social, empresarial, buenas prácticas ambientales, conservación y buen aprovechamiento de los entornos donde se realizan actividades de turismo de naturaleza y aventura.

4. Verificar que las empresas prestadoras de turismo de naturaleza y aventura mantengan actualizadas las respectivas pólizas de seguros necesarias para cubrir las actividades que desarrollan.

5. Realizar gestiones con las entidades correspondientes para que se capacite a los guías u orientadores turísticos en temas relacionados con seguridad personal y ambiental. 
6. Realizar acciones de prevención, control e impacto a los operadores turísticos de naturaleza y aventura en lo referente a la implementación de las normas técnicas sectoriales, respecto a las cuales se deben dejar los antecedentes documentales. Asimismo, confirmar que los guías que laboran en estas empresas cuenten con la certificación para realizar la actividad de orientador de turismo de naturaleza y aventura, pues los guías u orientadores turísticos que trabajen en estas empresas deben estar capacitados en atención de primeros auxilios y prevención de emergencias.

7. Diseñar planes de prevención y manejo de riesgos para las actividades de turismo de naturaleza y aventura que ofrece.

8. Dar a conocer al turista la importancia de no recibir de personas extrañas bebidas o alimentos que puedan causar alteraciones a su organismo. Se le recomienda al turista no caminar por lugares solitarios o desconocidos, que no sea ostentoso con el uso de joyas u objetos que lo conviertan en blanco fácil de la delincuencia, así como no suministrar información personal o financiera a desconocidos durante el desarrollo de las actividades de turismo de naturaleza y aventura. Así mismo, que antes de recibir productos de cualquier operador de turismo de naturaleza y aventura verifique que esté inscrito en el Registro Nacional de Turismo y que esté en un lugar visible al público (Decreto 504 de 1997, artículo 11), pues los prestadores de servicios turísticos están obligados a fijar copia auténtica del certificado de inscripción en un lugar del establecimiento visible al público.

9. Tener en cuenta la importancia de la conservación y la protección del medio ambiente, los recursos naturales y la prevención de amenazas y riesgos naturales en los sitios donde se realizan actividades de turismo de naturaleza y aventura. Esto implica advertir que no se deje basuras ni contaminantes en los entornos ecológicos donde se realicen las actividades de turismo de naturaleza y aventura.

10. Disponer que la autoridad ambiental de la jurisdicción realice actividades de prevención, control e impacto que permitan mitigar 
los efectos negativos que estas actividades tienen en los ecosistemas (se pueden apoyar con Policía de Protección Ambiental y Ecológica).

11. Diseñar estrategias encaminadas a concientizar a la comunidad aledaña a los entornos ecológicos y operadores turísticos, donde se resalte la importancia de la conservación y la protección del medio ambiente, los recursos naturales y la prevención de amenazas y riesgos naturales en los sitios donde se realizan actividades de turismo de naturaleza y aventura.

12. Dar orientaciones a la ciudadanía de no consumir bebidas embriagantes y/o sustancias psicoactivas durante el desarrollo de las actividades de turismo de naturaleza y aventura, con el fin de evitar accidentes que afecten su vida e integridad personal o la de las demás personas que se encuentren realizando esta clase de esparcimiento.

13. Realizar controles con personal idóneo a los elementos de movilidad (vehículos) y logística (elementos mecánicos y electrónicos utilizados en las actividades de turismo de naturaleza y aventura), con el fin de reducir las afectaciones a la vida y la seguridad personal de los practicantes de esta clase de actividades.

14. Desarrollar mesas de trabajo con funcionarios del Viceministerio de Turismo, empresas prestadoras de servicios y personal de policía de turismo, con el fin de elevar solicitudes ante las autoridades rectoras en materia de turismo. Al respecto, se evidencia que es necesario generar normatividades que regulen las modalidades de turismo de naturaleza y aventura que no se encuentran incluidas en las actuales normas sectoriales.

\section{Discusión}

El fenómeno turístico genera empleo, los gobiernos invierten gran cantidad de recursos económicos en logística e infraestructura, e incentivan a la sociedad a conservar sus costumbres y cultura autóctona, la cual es expuesta al sector turístico. Esto genera en el visitante conciencia ambientalista, la cual permite la conservación de los recursos naturales, y beneficia directamente a 
las empresas prestadoras de servicios turísticos y a los turistas. No obstante, la seguridad turística se ha visto afectada en los últimos años debido a la creciente inseguridad ciudadana y la violación de los Derechos Humanos, lo cual ha traído consecuencias negativas para algunos países, regiones y principales polos turísticos (González et al., 2017).

El turismo en todas sus expresiones genera contribuciones económicas a las poblaciones, y en este caso en especial a las del Departamento de Cundinamarca donde se desarrollan varias de estas actividades de aventura. Por lo tanto, se precisa indicar que este fenómeno recreativo y cultural afecta en grandes proporciones el entorno ambiental, como quiera que a mayor cantidad de visitantes en un área turística natural, es más difícil controlar los comportamientos de esa población flotante, lo cual dificulta en gran manera mantener los espacios en óptimas condiciones tanto para su conservación como para el disfrute de los demás turistas potenciales.

Durante el desarrollo del turismo de aventura se deben tener en cuenta diversos componentes que de alguna u otra forma podrían afectar la seguridad personal y situar en riesgo la vida e integridad física de las personas que realizan esta clase de actividades. Específicamente, se evidencian aspectos que han sido señalados como limitantes en el desarrollo y la competitividad del turismo de aventura en Colombia: (1) falta de adecuación de infraestructura, en el entendido de que si no se cuenta con la infraestructura y la logística necesarias, difícilmente se podrá dar un turismo de aventura seguro y de calidad. (2) Capacitación del personal de guías, pues se podría considerar que es un error permitir a un funcionario guiar actividades de turismo de aventura sin la idoneidad ni conocimiento básico, teniendo en cuenta que se manejan escenarios naturales con topografía y condiciones variables, aunado a esto el manejo de grupos de personas, situación que agravaría la situación que se llegase a presentar.

Asimismo, se evidenció la falta de articulación con prestadores de servicios de otras actividades de turismo de naturaleza, pues se requiere del compromiso de los prestadores y los operadores de servicios turísticos, sin importar que estas actividades de turismo de aventura no se encuentren dentro de los paquetes que ofrezcan al turista. También hace falta mejorar las condiciones técnicas 
para la prestación de servicios en el turismo de aventura, es por ello que se hace necesario y urgente generar los mecanismos y las herramientas que permitan a las personas que realizan actividades de turismo de aventura verificar que los elementos de seguridad personal que se utilizan cumplan las especificaciones técnicas y de uso de acuerdo con las normas técnicas sectoriales que fueron concebidas para garantizar la seguridad de los turistas.

Por último, se evidencia que los prestadores y operadores de servicios turísticos no aplican o cumplen las normas técnicas sectoriales desarrolladas para reglamentar las actividades de turismo de aventura. Este es un tema de notoria preocupación, toda vez que esta normatividad fue concienzudamente analizada y desarrollada por el Viceministerio de Turismo, con el fin de fijar y establecer criterios para la práctica de estas actividades, de manera que si no se aplica o se hace de manera incompleta, se pondría en riesgo la seguridad física de los participantes.

\section{Conclusiones}

A continuación se presentan los factores críticos que se identifican en el turismo de naturaleza y aventura en el Departamento de Cundinamarca y que requieren la intervención y el control por parte de la Policía Nacional:

- Muchas empresas ofertan el turismo de naturaleza y aventura sin cumplir los requisitos legales. La informalidad en la prestación de este tipo de turismo dificulta a las autoridades realizar mayores controles.

- Alta contaminación ambiental y daño en los ecosistemas al momento de realizar las prácticas de esta clase de turismo, y más cuando los operadores turísticos trabajan en la informalidad, pues pareciera no haber conciencia por la ecología ni respeto por la naturaleza; solo les interesa el factor económico.

- Carencia de pie de fuerza policial asignado a cumplir funciones de policía de turismo. Cundinamarca es un departamento con una gran extensión y numerosos municipios, pero solo cuenta con veinticuatro policías para realizar actividades de prevención y control 
en el turismo, lo cual facilita las cosas a los operadores turísticos informales.

- Otro factor crítico es el mal estado de los equipos que se utilizan para realizar esas actividades, teniendo en cuenta el poco control que realizan las autoridades y además la falta de preocupación de los turistas, quienes, como quedó evidenciado en el instrumento aplicado, no revisan ni indagan sobre el estado de dichos elementos.

- La falta de guías turísticos certificados por las autoridades competentes para realizar las actividades de turismo de naturaleza y aventura.

- La falta de medición de la capacidad de carga de los lugares donde se realiza el turismo de naturaleza y aventura, lo cual contribuye al deterioro de los lugares donde se desarrollan dichas actividades. Es decir, hay sobreexplotación de los lugares naturales.

- Falta de normatividad para regular todas las actividades de turismo de naturaleza y aventura, pues en la actualidad solo existen siete normas técnicas.

\section{Referencias}

Ashley, C., \& Roe, D. (2002). Involucramiento de la comunidad en el turismo de vida silvestre. International Institute for Environment and Development.

Ballesteros, M. A. (2004). Las estrategias de seguridad y defensa: Fundamentos de la estrategia para el siglo XXI [Monografía del CESEDEN, 67]. Ministerio de Defensa.

Brida, J., Monterubbianesi, P., \& Zapata, S. (2011). Impactos del turismo sobre el crecimiento económico y el desarrollo: El caso de los principales destinos turísticos de Colombia. Pasos, Revista de Turismo y Patrimonio Cultural, 9(2), 292.

Franco, S. S., \& Jaramillo, F. M. (2010). Modelo integral para el análisis y gestión del riesgo fisico preexistente en empresas contratantes de Estatal de Seguridad Ltda. [Tesis de maestría, Universidad Tecnológica de Pereira]. Repositorio UTP. http://repositorio.utp. edu.co/dspace/bitstream/handle/11059/1895/3631F825.pdf? sequence=1\&isAllowed $=y$

González, F. F., Hernández, T. C., Ganzo, O. J., \& Jimeno, E. R. (2017). La seguridad turística en México: Aportaciones para su configuración. Alternativas, 18(3), 84-93.

Huesear, A. (1993). Nuevo marco conceptual del turismo. Estudios Turisticos, (117), 23-48.

La Rotta, L. E. (2005). Consultor didáctico: Diccionario de seguridad. Metis. 
Ministerio de Comercio, Industria y Turismo. (2017). Plan Estratégico de Seguridad Turística. https://www.mincit.gov.co/getattachment/minturismo/calidad-y-desarrollo-sostenible/ seguridad-turistica/plan-estrategico-de-seguridad/plan-estrategico-de-seguridad-turistica/ plan-estrategico-de-seguridad-turistica.pdf.aspx

Rojas, R. N. (2011). Apoyo de la seguridad privada a la seguridad pública [Trabajo de especialización, Universidad Militar Nueva Granada]. Repositorio UMNG. https://repository.unimilitar.edu.co/bitstream/handle/10654/3454/RojasRojasNelsonHernando2011. pdf?sequence $=2 \&$ is Allowed $=y$

Organización Mundial del Turismo [OMT]. (2017). Código ético mundial para el turismo. https://www.oledays.es/que-es-el-codigo-etico-mundial-para-el-turismo.

Personal, M. W. (2002). Diccionario colegiado de Merriam-Webster (vol. 2). Merriam Webster.

Quezada, N. (2010). Metodología de la investigación: Estadistica aplicada a la investigación. Empresa Editorial Macro. 